Comment. Math. Helv. 75 (2000) 271-283

(C) 2000 Birkhäuser Verlag, Basel

$0010-2571 / 00 / 020271-13 \$ 1.50+0.20 / 0$

Commentarii Mathematici Helvetici

\title{
A note on degenerate corank-one singularities of integrable Hamiltonian systems
}

\author{
Nguyen Tien Zung
}

\begin{abstract}
We prove that, in a neighborhood of a corank-1 singularity of an analytic integrable Hamiltonian system with $n$ degrees of freedom, there is a locally-free analytic symplectic $\mathbb{T}^{n-1}$ action which preserves the moment map, under some mild conditions. This result allows one to classify generic degenerate corank-one singularities of integrable Hamiltonian systems. It can also be applied to the study of (non)integrability of perturbations of integrable systems.
\end{abstract}

Mathematics Subject Classification (2000). 58F07, 58F14.

Keywords. Integrable systems, torus actions, singularities.

\section{Introduction}

Let $\left(M^{2 n}, \omega\right)$ be a real analytic symplectic manifold, and $\mathbf{F}=\left(F_{1}, \ldots, F_{n}\right): M^{2 n} \rightarrow$ $\mathbb{R}^{n}$ be the moment map of an integrable Hamiltonian system on it. We will always assume $\mathbf{F}$ to be a proper map. By definition of integrability, the map $\mathbf{F}$ is regular almost everywhere. It is well-known that (a connected component of) the preimage of a regular value of $\mathbf{F}$ is an $n$-dimensional torus (called a Liouville torus), and in a neighborhood of this torus there is a free symplectic $\mathbb{T}^{n}$-action which preserves the moment map.

In general, near a singular level set of the moment map, such a $\mathbb{T}^{n}$-action no longer exists. However, one can hope that there still exists a torus action of smaller dimension. Indeed, in [9] we have shown, among other things, the existence of a locally free $\mathbb{T}^{n-k}$-action for a corank- $k$ nondegenerate singularity. Here a singularity is a germ of a tubular neighborhood of a singular level set of the moment map, and the corank is the maximal corank of the moment map at the points of the singular level set in question. The nondegeneracy condition is some natural condition, explained in detail in [9], and satisfied by most singularities of integrable systems known to us. In [3] we suggested that the existence of a locally free $\mathbb{T}^{n-k}$-action must be also true for degenerate singularities (under some very mild conditions), and showed how it is related to the (non)integrability of 
perturbations of integrable systems. In the second part of [9] we showed how to use these torus actions to define characteristic classes, which are global topological invariants of integrable Hamiltonian systems.

The main aim of this note is to give a proof of the existence of locally-free $\mathbb{T}^{n-1}$ actions for (degenerate) corank-1 singularities of integrable Hamiltonian systems. Let us now formulate the main theorem. As above, let $\mathbf{F}=\left(F_{1}, \ldots, F_{n}\right): M^{2 n} \rightarrow$ $\mathbb{R}^{n}$ be a proper analytic moment map, and assume that $N=\mathbf{F}^{-1}(0)$ is a connected compact singular level set of corank 1 . It means that the rank of the (differential of the) map $\mathbf{F}$ at any point of $N$ is at least $(n-1)$, and there is a point $x \in N$ where the rank of $\mathbf{F}$ is $(n-1)$. Since $N$ is preserved by the Poisson $\mathbb{R}^{n}$-action of the moment map $\mathbf{F}$, and the dimension of each orbit of this action in $N$ is at least $n-1$ because the rank of $\mathbf{F}$ is at least $n-1$, the dimension of $N$ is at least $n-1$. Since the rank of $\mathbf{F}$ is at least $n-1$ everywhere on $N$, the dimension of $N$ is at most $2 n-(n-1)=n+1$. We have:

Theorem 1.1. If $\operatorname{dim} N=n-1$, or $\operatorname{dim} N=n$ and $N$ contains a regular point, then in a neighborhood of $N$ in $\left(M^{2 n}, \omega\right)$ there is an analytic locally-free symplectic $\mathbb{T}^{n-1}$-action which preserves the moment map and which is free on Liouville tori. If $\operatorname{dim} N=n$ and all the points of $N$ are singular, then such a $\mathbb{T}^{n-1}$-action exists in either a neighborhood of $N$ or a finite covering of a neighborhood of $N$.

The case $\operatorname{dim} N=n+1$ is too degenerate, and is ruled out from the above theorem. For example, consider an integrable system with two degrees of freedom and assume for example that the isoenergy level set $Q=\left\{F_{1}=0\right\}$ is regular. Then by changing $F_{2}$ by $F_{2}^{\prime}=F_{1} F_{2}, Q$ becomes a corank-1 singular level set. On the other hand, according to a result of Fomenko (see e.g. [4]), $Q$ can be any graph-manifold and does not admit any locally-free $\mathbb{S}^{1}$-action in general.

Theorem 1.1 will follow from the following "local" result. A part of this result was obtained in collaboration with Alexey Bolsinov.

Theorem 1.2. Let $\mathcal{O}$ be a compact orbit of dimension $n-1$ of the Poisson $\mathbb{R}^{n}$ action of the analytic moment map $\mathbf{F}:\left(M^{2 n}, \omega\right) \rightarrow \mathbb{R}^{n}$ of an integrable Hamiltonian system. Assume that the level set of the moment map which contains this orbit has dimension at most $n$. Then in a neighborhood of $\mathcal{O}$ there is a locally free analytic symplectic $\mathbb{T}^{n-1}$ action which preserves $\mathcal{O}$ and the moment map. If $\mathcal{O}$ is not of nondegenerate elliptic type, then this $\mathbb{T}^{n-1}$-action is also unique, up to homomorphisms of $\mathbb{T}^{n-1}$.

The nondegenerate ellipticity condition in the above theorem can be formulated as follows: let $D^{n+1}$ be a sufficiently small $(n+1)$-dimensional ball in $M^{2 n}$ which intersects $\mathcal{O}$ transversally. Then $\mathcal{O}$ is called of nondegenerate elliptic type if there is a system of coordinates $\left(q_{1}, \ldots, q_{n+1}\right)$ on $D^{n+1}$ such that the moment map $\mathbf{F}$ restricted to $D^{n+1}$ can be written as a map of $n$ variables $\left(q_{1}, \ldots, q_{n-1}, q_{n}^{2}+q_{n+1}^{2}\right)$. 
If $\mathcal{O}$ is of nondegenerate elliptic type, then there is not only a $\mathbb{T}^{n-1}$-action, but also a $\mathbb{T}^{n}$-action in a neighborhood of $\mathcal{O}$ (see [9]).

The rest of this note is organized as follows: In Section 2 we give a lemma about an interesting property of the automorphisms of complex curve singularities. This lemma is used in the proof, given in Section 3 and Section 4, of Theorem 1.2 and Theorem 1.1 respectively. In Section 5 we will discuss generic degenerate singularities of corank 1 of integrable systems.

This note arises partially from some discussions with Alexey Bolsinov about local circle actions during his visit to Montpellier in May 1996, and I would like to thank him. I would like to thank the referee for his critical remarks.

\section{Automorphisms of curve singularities}

Let $f:\left(\mathbb{C}^{2}, 0\right) \rightarrow(\mathbb{C}, 0)$ be a germ of a non-zero analytic function of two complex variables, $f(0)=0$. Assume that $0 \in \mathbb{C}^{2}$ is a singular point of $f$, which may be isolated or not. Let $B \subset \mathbb{C}^{2}$ be a small ball centered at the origin, in which $f$ is defined and has 0 as the only critical value. For $\epsilon \in \mathbb{C}, \epsilon \neq 0$ but near to 0 , denote by $S_{\epsilon}$ the Milnor fiber $S_{\epsilon}=f^{-1}(\epsilon) \cap B$. Let $\phi:\left(\mathbb{C}^{2}, 0\right) \rightarrow\left(\mathbb{C}^{2}, 0\right)$ be a germ of an analytic inversible map which preserves $f$. Then $\phi$ maps $S_{\epsilon}^{\prime}$ into $S_{\epsilon}$, where $S_{\epsilon}^{\prime}=B^{\prime} \cap f^{-1}(\epsilon), B^{\prime}$ being a ball in $\mathbb{C}^{2}$ such that $0 \in B^{\prime} \subset B$ and $\phi\left(B^{\prime}\right) \subset B$. Since $S_{\epsilon} \backslash S_{\epsilon}^{\prime}$ is a union of cylinders, the map $\left.\phi\right|_{S_{\epsilon}^{\prime}}: S_{\epsilon}^{\prime} \rightarrow S_{\epsilon}$ may be extended to a map from $S_{\epsilon}$ to itself, which is not unique but homotopically unique (for $|\epsilon|$ small enough). We will denote this map by $\phi_{\epsilon}: S_{\epsilon} \rightarrow S_{\epsilon}$.

Lemma 2.1. There is a positive integer $m$ such that $\phi_{\epsilon}^{m}$, the $m$-time iteration (i.e. power of order $m$ ) of $\phi_{\epsilon}$, is homotopic to identity in $S_{\epsilon}$ (for $|\epsilon|$ small enough).

Proof. We will desingularize the singular fiber $S_{0}=f^{-1}(0) \cap B$ by blow-ups. As a result, $B$ will be changed to $B \# k \overline{\mathbb{C} P} P^{2}$ for some natural number $k$, with an analytic $\operatorname{map} \bar{f}: B \# k \overline{\mathbb{C} P} \bar{P}^{2} \rightarrow \mathbb{C}$ (which is the pull-back of $f$ ) such that the preimage $\bar{f}^{-1}(0)$ of 0 consists of the desingularization $\bar{S}_{0}^{\prime}$ of $S_{0}$ and the exceptional divisors, and the analytic set $\bar{f}^{-1}(0)$ (without counting multiplicities) contains no other singular points than the nodes, i.e. double points. Since $\phi$ preserves $f$, it may be lifted to a local automorphism from $B \# k \overline{\mathbb{C P}} P^{2}$ to itself which preserves $\bar{f}$. Replacing $\phi$ by $\phi^{h}$ for some $h \in \mathbb{Z}_{+}$if necessary, we can assume that $\bar{\phi}$ preserves each exceptional divisor and each connected component of $\bar{S}_{0}$. Hence $\bar{\phi}$ also preserves each double point of $\bar{f}^{-1}(0)$. The Lemma is almost trivial if $f^{-1}(0)$ has only a node singularity itself, since then $S_{\epsilon}$ is a cylinder. Assume now that $f^{-1}(0)$ has a more complicated singularity at 0 . Then there is an exceptional divisor $D \subset \bar{f}^{-1}(0)$ which contains at least 3 double points of $\bar{f}^{-1}(0)$. An holomorphic automorphism of $\mathbb{C} P^{1}$ with 3 fixed points is identity. Thus $\bar{\phi}$ is identity on $D$. Consider another exceptional 
divisor $D_{1}$ in $\bar{f}^{-1}(0)$, which intersects $D$ at a double point $z$. The function $\bar{f}$ near $x$ can be written in the form $\bar{f}=x^{p} y^{q}$, where $(x, y)$ is a local system of complex coordinates such that $x=0$ on $D$ and $y=0$ on $D_{1}, p$ and $q$ are multiplicities of $\bar{f}=0$ on $D$ and on $D_{1}$ respectively. since $\bar{\phi}$ preserves $\bar{f}, D$ and $D_{1}$, we have that $\bar{\phi}(x, y)=(x(a+\ldots), y(b+\ldots))$ with numbers $a, b \in \mathbb{C}$ such that $a^{p} b^{q}=1$. Since $\bar{\phi}$ is identity on $D$, we have $a=1$. By replacing $\phi$ by $\phi^{q}$, we can assume that $b=1$. In particular, the differential of (some power of) $\bar{\phi}$ at $z$ is identity. Since, beside $z, D_{1}$ contains at least one other double point of $\bar{f}^{-1}(0)$, which will be also fixed by $\bar{\phi}$, it follows that (a power of) $\bar{\phi}$ is identity on $D_{1}$. (Recall that if a holomorphic automorphism $\psi$ of $\mathbb{C} P^{1}$ fixes the points $0, \infty$ then it will be of the type $\psi(y)=b y$. If in addition the differential of $\psi$ at 0 is identity, then $b=1$ and $\psi$ is identity). The set of exceptional divisors in $\bar{f}^{-1}(0)$ forms a tree, if we replace each exceptional divisor by a vertex and each double point by an edge. Hence, by induction, we obtain that a power of $\bar{\phi}$, say $\bar{\phi}^{m}$, is identity on the union of exceptional divisors, and has differential equal to identity at every double point. It implies that $\bar{\phi}_{\epsilon}^{m}$ is $C^{0}$-close to identity on $\bar{S}_{\epsilon}=\bar{f}^{-1}(\epsilon)$, and hence homotopic to identity, and we are done (since $\bar{S}_{\epsilon}$ is the same as $S_{\epsilon}$ ). To see it more clearly, at each double point $z \in \bar{f}^{-1}(0)$ let $B_{z}$ be a small ball at $z$. Then the spheres $\partial D_{z}$ cut $\bar{S}_{\epsilon}$ into pieces, on each of which $\bar{\phi}_{m}^{m}$ will be clearly close enough to identity. In particular, since the differential of $\bar{\phi}^{m}$ at double points is identity, $\bar{\phi}^{m}$ does not "mix" the local leaves of the local finite covering of the pieces of $\bar{S}_{\epsilon}$ over punctured exceptional divisors and punctured components of $\bar{S}_{0}$.

\section{Local construction of the torus action}

In this section we will prove Theorem 1.2. Let $\mathcal{O}$ be a compact $(n-1)$-orbit of the $\mathbb{R}^{n}$-Poisson action of the moment map. Then it is well-known that $\mathcal{O}$ is an $(n-1)$ dimensional torus, since it is a compact quotient of $\mathbb{R}^{n}$ by some subgroup. By a linear transformation of the moment map $\mathbf{F}=\left(F_{1}, \ldots, F_{n}\right)$, we can assume that the isotropy group of the $\mathbb{R}^{n}$-Poisson action on $\mathcal{O}$ consists of the elements of the type $\left(p_{1}, \ldots, p_{n-1}, x_{n}\right)$ where $p_{i}^{\prime} s$ are integers and $x_{n}$ is an arbitrary real number. It means in particular that $d F_{n}=0$ on $\mathcal{O}$, and the actions of the Hamiltonian vector fields $X_{F_{1}}, \ldots, X_{F_{n-1}}$ of the functions $F_{1}, \ldots, F_{n-1}$ are independent and periodic of period 1 on $\mathcal{O}$.

Without the loss of generality, we can assume that the moment map has value 0 on the orbit $\mathcal{O}: \mathbf{F}(\mathcal{O})=(0, \ldots, 0)$. Let $D^{n+1}$ be a small $(n+1)$-dimensional ball in $M^{2 n}$, intersecting $\mathcal{O}$ transversally at a given point $x \in \mathcal{O}$. Then $\left(F_{1}, \ldots, F_{n-1}\right)$ restricted to $D^{n+1}$ will be a regular map, which divides $D^{n+1}$ into an $(n-1)$ dimensional family of 2-dimensional disks $D_{\mathbf{f}}^{2}=\left\{\left(F_{1}, \ldots, F_{n-1}\right)=\mathbf{f}=\left(f_{1}, \ldots, f_{n-1}\right)\right\}$. The local symplectic reduction with respect to the Poisson $\mathbb{R}^{n-1}$-action generated by $\left(F_{1}, \ldots, F_{n-1}\right)$ induces on $D^{n+1}$ a regular Poisson structure with symplectic 
leaves $\left(D_{\mathbf{f}}^{2}, \omega_{\mathbf{f}}\right)$.

Fix a non-zero $(n-1)$-tuple of integers $\mathbf{p}=\left(p_{1}, \ldots, p_{n-1}\right)$. The Poisson $\mathbb{R}^{n-1}$ action (generated by $\left.\left(F_{1}, \ldots, F_{n-1}\right)\right)$ is identity on $\mathcal{O}$ at the multi-time $\mathbf{p}$. It follows that, for a point $y \in D^{n+1}$, there is a unique multi-time $\mathbf{p}(y)$ close to $\mathbf{p}$ and depending analytically on $y$, such that the Poisson $\mathbb{R}^{n-1}$-flow moves $y$ to a point $z \in D^{n+1}$ at multi-time $\mathbf{p}(y)$. The (analytic) mapping $y \mapsto z$ is called the Poincaré map for the multi-time $\mathbf{p}$, in analogy with the Poincaré map for a periodic orbit of a dynamical system. For a fixed (nonzero integral) multi-time p, the Poincaré map is a germ of an analytic diffeomorphism from $\left(D^{n+1}, x\right)$ to itself, which preserves the moment map $\mathbf{F}$ and the Poisson structure of $D^{n+1}$. Denote this Poincaré map by $\phi_{\mathbf{p}}$. Recall that the level set of $\mathbf{F}$ which contains $\mathcal{O}$ is of dimension at most $n$ by assumptions. It means that $F_{n}$ is not a constant function on the disk $D_{0}^{2}$.

Lemma 3.1. There is a positive integer $m$ such that $\phi_{\mathbf{p}}^{m}$ (the $m$-time iteration of $\phi_{\mathbf{p}}$ ) is the time-1 map of an analytic Hamiltonian vector field on $D^{n+1}$ (of an analytic Hamiltonian function $G$ on $D^{n+1}$, with respect to the induced Poisson structure), which is tangent to the level sets of the function $F_{n}$ in $D^{n+1}$. If $\mathcal{O}$ is not of nondegenerate elliptic type, then this Hamiltonian vector field is also unique (for each admissible $m$ ).

Proof. Complexifying the map $\mathbf{F}$ restricted to $D^{n+1}$, we get a map $\mathbf{F}=\left(F_{1}, \ldots\right.$, $\left.F_{n-1}, F_{n}\right): D_{\mathbb{C}}^{n+1} \rightarrow \mathbb{C}^{n}$. $\phi_{\mathbf{p}}$ becomes a holomorphic automorphism of $D_{\mathbb{C}}^{n+1}$ preserving $\mathbf{F}$. Since $F_{n}$ is not identically zero on $D_{0}^{2}$, Lemma 2.1 implies that there is a positive integer $m$ such that $\phi_{\mathbf{p}}^{m}$ is homotopic to identity on each Milnor fiber (i.e. local regular level set of $\mathbf{F}) S_{\mathbf{f}, \epsilon}=\left\{F_{1}=f_{1}, \ldots, F_{n-1}=f_{n-1}, F_{n}=\epsilon\right\} \cap D_{\mathbb{C}}^{n+1}$ $\left(\left|f_{1}\right|, \ldots,\left|f_{n-1}\right|,|\epsilon|\right.$ small enough). Let $y \in S_{\mathbf{f}, \epsilon}$ be a point lying in a Milnor fiber. By starting at $y$ and following a homotopy from identity to $\phi_{\mathbf{p}}^{m}$, we will get a path $\gamma_{y}$ from $y$ to $\phi_{\mathbf{p}}^{m}(y), \gamma_{y} \subset S_{\mathbf{f}, \epsilon}$. We will assume that $F_{n}$ restricted to $D_{0}^{2}=\left\{F_{1}=\right.$ $\left.\ldots=F_{n-1}=0\right\}$ has 0 as the only singular value, and the preimage $F_{n}^{-1}(0)$ (on $D_{0}^{2}$ ) is a singular curve with a singularity (at point $x$ ) which is not a node (i.e. double-point) singularity. (The case when $x$ is a double point, or a regular point, is similar and simpler). Then $\gamma_{y}$ has two remarkable properties: 1) It is uniquely defined up to homotopies rel. $y, \phi_{\mathbf{p}}^{m}(y)$. 2) If $y$ is real, i.e. $y \in S_{\mathbf{f}, \epsilon} \cap D^{n+1}$, then $\gamma_{y}$ is homotopic rel. $y, \phi_{\mathbf{p}}^{m}(y)$ to a real path, i.e. to a path lying in $S_{\mathbf{f}, \epsilon} \cap D^{n+1}$. Indeed, the fundamental group $\pi_{1}\left(S_{\mathbf{f}, \epsilon}\right)$ is a free group of at least 2 generators, and the center of $\pi_{1}\left(S_{\mathbf{f}, \epsilon}\right)$ is trivial. But if $\gamma_{y}$ and $\gamma_{y}^{\prime}$ are two different paths obtained by two different homotopies from identity to $\phi_{\mathbf{p}}^{m}$, then $\gamma_{y}^{-1} \gamma_{y}^{\prime}$ lies in the center of $\pi_{1}\left(S_{\mathbf{f}, \epsilon}\right)$. Hence $\gamma_{y}^{-1} \gamma_{y}^{\prime}$ is homotopically trivial, and $\gamma_{y}$ is homotopic to $\gamma_{y}^{\prime}$ rel. $y, \phi_{\mathbf{p}}^{m}(y)$. For the second property, observe that $y$ and $\phi_{\mathbf{p}}^{m}(y)$ lie in a same connected component of $S_{\mathbf{f}, \epsilon} \cap D^{n+1}$ (or at least we can assume so by taking a multiple of $n$ ). Denote this component by $\alpha$, and a path connecting $y$ to $\phi_{\mathbf{p}}^{m}(y)$ lying in this component by $\beta_{y}$. If $\alpha$ is closed (the degenerate elliptic case) then it 
gives a simple non-trivial element in $\pi_{1}\left(S_{\mathbf{f}, \epsilon}, y\right)$, which commutes with $\gamma_{y}^{-1} \beta_{y}$ in $\pi_{1}\left(S_{\mathbf{f}, \epsilon}, y\right)$, because $\phi_{\mathbf{p}}^{m}$ preserves $\alpha$ and is homotopic to identity. It follows that $\gamma_{y}^{-1} \beta_{y}$ is homotopic to a multiple of $\alpha$. In particular, $\gamma_{y}^{-1} \beta_{y}$ is homotopic to a real path, and so is $\gamma_{y}$ rel. $y, \phi_{\mathbf{p}}^{m}(y)$. If $\alpha$ is not closed (degenerate hyperbolic case) then $\alpha$ has two boundary points, say $z_{1}$ and $z_{2}$. Then $\phi_{\mathbf{p}}^{m}$ preserves $z_{1}$ and $z_{2}$, and the homotopy from identity to $\phi_{\mathbf{p}}^{m}$ can be chosen so that each element in this homotopy also preserves $z_{1}$ and $z_{2}$. It follows that $\gamma_{y}^{-1} \beta_{y}$ is homotopically trivial in this case.

Let $\theta$ be a real analytic 1 -form on $D^{n+1}$ such that $d \theta_{\mathbf{f}}=\omega_{\mathbf{f}}$ (the reduced symplectic form) on each $D_{\mathbf{f}}^{2}=\left\{F_{1}=f_{1}, \ldots, F_{n-1}=f_{n-1}\right\} \cap D^{n+1}$, where $\theta_{\mathbf{f}}$ is the pull-back of $\theta$ to $D_{\mathbf{f}}^{2}$. since $\phi_{\mathbf{p}}^{n *}\left(\omega_{\mathbf{f}}\right)=\omega_{\mathbf{f}}$, we have that $d\left(\phi^{n *} \theta_{\mathbf{f}}-\theta_{\mathbf{f}}\right)=0$, and there is an analytic function $R(y)$ such that $d R_{\mathbf{f}}(y)=\phi_{\mathbf{p}}^{n *} \theta_{\mathbf{f}}-\theta_{\mathbf{f}}$, where $R_{\mathbf{f}}=\left.R\right|_{D_{\mathbf{f}}^{22}}$. Define (for $y$ lying in a Milnor fiber)

$$
G(y)=\int_{\gamma_{y}} \theta-R(y)
$$

The properties of the path $\gamma_{y}$ discussed above imply that $G(y)$ can be extended to a single-valued holomorphic function in $D_{\mathbb{C}}^{n+1}$, which has real value when $y$ is real. It is not difficult to verify directly that $G(y)$ is the Hamiltonian function giving the Hamiltonian flow on $D^{n+1}$ that we are looking for.

As for the uniqueness, let us suppose that there are two different Hamiltonian vector fields on $D^{n+1}$ which satisfy the above conditions. Then they must commute, because they are tangent to each other. Their difference will be a non-zero Hamiltonian vector field, whose time-1 map is identity (i.e. a periodic Hamiltonian vector field), and it follows that in this case $\mathcal{O}$ is of nondegenerate elliptic type.

We will suppose that $\mathcal{O}$ is not of nondegenerate elliptic type (the nondegenerate elliptic case has been studied in [9]). It is clear that the function $G(y)$ constructed above is invariant on the level sets of the moment map $\mathbf{F}$ on $D^{n+1}$. We can assume that $d G(x)=0$ on $D^{n+1}$. Due to the uniqueness of the Hamiltonian vector field in the above lemma, $G$ is uniquely defined, modulo a function of $F_{1}, \ldots, F_{n-1}$. Notice that the Poincaré maps for different (integral) multitimes commute, and that $\phi_{\mathbf{p}}^{m}=\phi_{m \mathbf{p}}$. Due to its uniqueness and the fact that it's invariant on the level sets of $F_{n}, Q$ is invariant under the Poincaré map of any integral multi-time. Thus we can extend $G$ invariantly (with respect to the Hamiltonian vector fields $\left.X_{F_{1}}, \ldots, X_{F_{n-1}}\right)$ to obtain an analytic function in a neighborhood of the orbit $\mathcal{O}$, which we will denote again by $G$.

Put $G_{1}=m\left(p_{1} F_{1}+\ldots+p_{n-1} F_{n-1}\right)+G$ (where $\mathbf{p}=\left(p_{1}, \ldots, p_{n-1}\right)$ is the multitime). Then $G_{1}$ is an analytic function in a neighborhood of $\mathcal{O}$, whose Hamiltonian vector field $X_{G_{1}}$ has the following property: the time-1 map of its flow, denoted by $\psi$, has $x$ as a fixed point, and sends any point $y \in D^{n+1}$ to a point $y^{\prime}$ near $y$, whose projection to $D^{n+1}$ via the local integral curves of the vector fields $X_{F_{1}}, \ldots, X_{F_{n-1}}$ is again $y$. It follows that $\psi$ is also the time- 1 map of the flow of an analytic vector 
field $X_{1}$, which is commuting with the Poisson $\mathbb{R}^{n}$-action of the integrable system, and which is linearly dependent of $X_{F_{1}}, \ldots, X_{F_{n-1}}: X_{1}=c_{1} X_{F_{1}}+\ldots+c_{n-1} X_{F_{n-1}}$, where $c_{1}, \ldots, c_{n-1}$ are analytic functions which vanish at $x$. The vector field $X=$ $X_{G_{1}}-X_{1}$ will be a periodic Hamiltonian vector field which preserves the moment map, and on $\mathcal{O}$ it coincides with $m\left(p_{1} X_{F_{1}}+\ldots+p_{n-1} X_{F_{n-1}}\right)$. So it gives a locally free Hamiltonian $\mathbb{S}^{1}$-action which preserves the moment map.

Take $(n-1)$ linearly independent multi-times. They will give $(n-1)$ commuting actions of $\mathbb{S}^{1}$ by the above process, and together they will form a locally-free Hamiltonian $\mathbb{T}^{(n-1)}$-action in a neighborhood of $\mathcal{O}$, which preserves the moment map.

About the uniqueness: if we don't have the uniqueness of the $\mathbb{T}^{n-1}$-action (up to morphisms of $\mathbb{T}^{n-1}$ ), then because of the commutativity, we will have a Hamiltonian $\mathbb{T}^{n}$-action (which is locally free almost everywhere). It is easy to see that in this situation $\mathcal{O}$ must be of nondegenerate elliptic type.

\section{Proof of Theorem 1.1}

Let $N=\mathbf{F}^{-1}(0)$ be a connected singular level set of dimension at most $n$, as in the assumptions of Theorem 1.1. Due to the analycity and the invariance with respect to the Poisson action, $N$ has a smooth stratification, whose strata are invariant under the Poisson $\mathbb{R}^{n}$-action. Since $N$ is a corank- 1 singularity, the orbits of the Poisson action in it have dimension at least $n-1$. Thus the strata of $N$ are of dimension $n$ or $n-1$.

There are three possible cases: 1) $N$ is of dimension $(n-1)$;2) $N$ is of dimension $n$ and contains a regular point (i.e. a point where the rank of the moment map is $n)$; 3) $N$ is of dimension $n$ and does not contain a regular point. In the first case, where $\operatorname{dim} N=n-1, N$ is smooth since it cannot contain a stratum of smaller dimension. It follows that $N$ is a compact orbit of dimension $(n-1)$ of the Poisson action. The existence of a torus action in a neighborhood of $N$ in this case is assured by Theorem 1.2.

Consider now the second case, when $N$ contains a regular point $x$. Let $\mathcal{O}(x)$ denote the orbit via $x$ of the Poisson $\mathbb{R}^{n}$-action. Since $x$ is regular, $\mathcal{O}(x)$ is of dimension $n$. And since $N$ is singular, $\mathcal{O}(x)$ cannot be a closed orbit. It follows that the boundary of $\mathcal{O}(x)$ will contain an orbit of smaller dimension of the Poisson action, which we will denote by $\mathcal{O}(y)$. Since $N$ does not contain strata of dimension smaller than $(n-1)$, it follows that $\mathcal{O}(y)$ must be closed, of dimension $(n-1)$, and is a connected component of the boundary of $\mathcal{O}(x)$ in $N$. In the literature, $\mathcal{O}(y)$ is called a whiskered torus (with $\mathcal{O}(x)$ being a part of the whisker). Theorem 1.2 shows that there is a locally free Hamiltonian $\mathbb{T}^{n-1}$-action in a neighborhood of $\mathcal{O}(y)$, which preserves the moment map. We can assume that $x$ is close enough to $\mathcal{O}(y)$ to lie in this neighborhood. The $\mathbb{T}^{n-1}$-action is generated by $(n-1)$ independent periodic Hamiltonian functions, say $H_{1}, \ldots, H_{n}$. These functions are 
invariant on the level sets of the moment map (in a neighborhood of $\mathcal{O}(y)$ ). Since $x$ is a regular point of the moment map, in a small neighborhood of $x$ we have $\left(H_{1}, \ldots, H_{n-1}\right)=\mathbf{H}\left(F_{1}, \ldots, F_{n}\right)$ where $\mathbf{H}$ is an analytic function from a domain of $\mathbb{R}^{n}$ to $\mathbb{R}^{n-1}$. But then the formula $\left(H_{1}, \ldots, H_{n-1}\right)=\mathbf{H}\left(F_{1}, \ldots, F_{n}\right)$ will give us $(n-1)$ analytic functions well-defined in a neighborhood of $N$. The time-1 maps of their Hamiltonian vector fields are identity in a neighborhood of $\mathcal{O}(y)$, and hence, by analytic continuation, are identity in a neighborhood of $N$. Since they are obviously commuting, they give us a Hamiltonian $\mathbb{T}^{n-1}$-action which preserves the moment map. The fact that this action is locally-free follows easily from the non-ellipticity of $N$. (See e.g. [2] and references therein for Hamiltonian torus actions).

Consider now the last case, when $\operatorname{dim} N=n$ and every point of $N$ is singular. We divide this case into two subcases:

Subcase 1. $N$ does not contain any compact $(n-1)$-dimensional orbit of the Poisson action. In this case all the level sets of the moment map in a neighborhood of $N$ (including $N$ itself), are smooth. Indeed, if $N$ is not smooth then it will contain a stratum of dimension $n-1$, and this stratum will be a compact orbit of the Poisson action. We will denote by $\mathcal{U}(N)$ a sufficiently small neighborhood of $N$. At a point $x \in N$, we can assume that $d F_{1} \wedge \ldots \wedge d F_{n-1}(x) \neq 0$. Then $d F_{1} \wedge \ldots \wedge d F_{n-1} \neq 0$ everywhere on $N$, since otherwise the set where it vanishes would contain a compact orbit of the Poisson action. Thus we can assume that $d F_{1} \wedge \ldots \wedge d F_{n-1} \neq 0$ everywhere in $\mathcal{U}(N)$. The set of points in $\mathcal{U}(N)$, where the rank of $\mathbf{F}$ is $(n-1)$, is an analytic set which will be denoted by $K$. The set of points in $K$ whose local level sets are still smooth is another analytic set, denoted by $K^{\prime}$. We have $N \subset K^{\prime} \subset K$, and we want that $K^{\prime}=K$. If $K^{\prime} \neq K$, then it is easy to see that there is a projective element $\left(f_{1}: \ldots: f_{n-1}\right) \in \mathbb{R} P^{n-1}$ such that the set $\left\{y \in K, F_{1}(y): \ldots: F_{n-1}(y)=f_{1}: \ldots: f_{n-1}\right\}$ is an analytic set of dimension $n$, not contained in $K^{\prime}$, and that $N$ is not a connected component of this set. Since $N$ is contained in this set, a smooth stratification of this set will have a stratum lying in $N$ which is not $N$. And since this stratification is invariant under the Poisson action, it follows that $N$ contains a closed subset of smaller dimension, which is invariant under the Poisson $\mathbb{R}^{n}$ action. In particular, $N$ will contain a compact orbit of dimension $(n-1)$, which is contradictory to our assumptions. Thus, in a neighborhood $\mathcal{U}(N)$ of $N$, we have a foliation whose leaves are smooth analytic sets which are connected components of the level sets of the moment map. Due to the analycity of the moment map, this foliation is an analytic foliation (i.e. locally, in a small neighborhood of any point, the moment map can be changed by a regular analytic map which defines the same level sets). This foliation has compact leaves (at least near $N$ ), and the generic leaf is an $n$ dimensional torus. Consider the holonomy of this foliation at a point $x \in N$. This holonomy preserves the (differential of the) moment map. Since the moment map has rank $(n-1)$ at $x$, it follows easily that the holonomy is either trivial or $\mathbb{Z}_{2}$. If the case of trivial holonomy, we have a topologically trivial foliation, and the classical 
Arnold-Liouville theorem (or rather the proof of it) gives us a free Hamiltonian $\mathbb{T}^{n}$-action in a neighborhood of $N$. In the case of holonomy $\mathbb{Z}_{2}$, let $\overline{\mathcal{U}(N)}$ be the corresponding 2-covering of $\mathcal{U}(N) . \overline{\mathcal{U}(N)}$ is a topologically trivial foliation by Lagrangian tori, and we have a Hamiltonian $\mathbb{T}^{n}$-action on it, whose moment map (which vanishes on the 2-covering of $N$ ) will be denoted by $\left(H_{1}, \ldots, H_{n}\right)$. $H_{1}, \ldots, H_{n}$ are called action variables. The space of variables $\left(H_{1}, \ldots, H_{n}\right)$ has a natural linear structure, and contains a natural integral lattice of dimension $n$ (the points of this lattice corresponds to Hamiltonian functions whose Hamiltonian flows preserves the Lagrangian tori and are periodic of period $1 / k$ for some natural number $k$ ). The involution on $\overline{\mathcal{U}(N)}$ induces a linear involution on the space of action variables $\left(H_{1}, \ldots, H_{n}\right)$, which preserves the integral lattice. The moment map $\mathbf{F}$ can be considered as an analytic map of variables $\left(H_{1}, \ldots, H_{n}\right)$, and at 0 this map is of rank $(n-1)$. The linear part of this map at 0 is also of rank $(n-1)$ and it preserved by the involution. It follows easily that this involution fixes an integral sublattice of dimension $(n-1)$. This sublattice will give us a Hamiltonian $\mathbb{T}^{n-1}$-action in a neighborhood of $N$.

Subcase 2. Some (or all) orbits of the Poisson $\mathbb{R}^{n}$-action in $N$ are compact (of dimension $n-1$ ). Theorem 1.2 shows the existence and uniqueness of locallyfree moment-map-preserving Hamiltonian $\mathbb{T}^{n-1}$ actions near these compact orbits. We want to glue these actions together, and extend them analytically, to obtain a locally free $\mathbb{T}^{n-1}$ action in a neighborhood of $N$. There are two possible kinds of obstructions to doing so:

1. Divergence: while trying to extend the action variables (i.e. the moment map of a $\mathbb{T}^{n-1}$ action) from a neighborhood of a compact orbit in $N$ to a neighborhood of $N$, some action variable may diverge (i.e. cannot be extended analytically) somewhere. The set of points in $N$ where at least one of the action variables diverges is closed (due to the uniqueness of the $\mathbb{T}^{n-1}$ action wherever it's defined, and hence the uniqueness of the action variables up to an affine transformation), and is of dimension smaller than $n$ in $N$. This set is also invariant under the Poisson $\mathbb{R}^{n}$-action. But then this set consists of compact orbits of the Poisson action, and hence by Theorem 1.2 the $\mathbb{T}^{n-1}$ action is well-defined there. Thus in fact there is no divergence.

2. Non-trivial holonomy: We can always assume that the locally-defined $(n-1)$ action variables above vanish on $N$. The problem is that, after going around a homotopically non-trivial path in $N$, the action variables are changed by a linear automorphism of $\mathbb{R}^{n-1}$ which may be different from identity. We will show that the holonomy group is finite. Then we can take a corresponding finite covering of a neighborhood of $N$ to kill this holonomy group. When the holonomy group is trivial, the $(n-1)$ action variables are well-defined, so their corresponding $\mathbb{T}^{n-1}$ action is well defined in a neighborhood of $N$. To show that the holonomy group is finite, we will use the fact that the moment map is proper. Take a point $x$ lying in a smooth part of $N$, and let $D^{n}$ be a small analytic disk transversal to $N$ at $x$. Restricting the moment map $\mathbf{F}$ to $D^{n}$, we get a local analytic map $\mathbf{F}:\left(D^{n}, x\right) \rightarrow$ 
$\left(\mathbb{R}^{n}, 0\right)$. Complexify this map to get a holomorphic map $\mathbf{F}_{\mathbb{C}}:\left(D_{\mathbb{C}}^{n}, x\right) \rightarrow\left(\mathbb{C}^{n}, 0\right)$. The number of points in the preimage of $\mathbf{F}_{\mathbb{C}}$ of a generic point close enough to 0 in $\left(\mathbb{C}^{n}, 0\right)$ will be called the multiplicity of $\mathbf{F}$ at $x$. Since $\mathbf{F}$ is proper, this multiplicity is a well defined finite positive integer (depending on $x$ ). Let $\gamma$ be a simple piece-wise smooth closed curve in $N$ starting at $x$. Then for each $y$ in $D_{\mathbb{C}}^{n}$ close enough to $x$ there exists a curve $\Gamma$ lying in a small tubular neighborhood of $\gamma$ of a complexification of $M^{2 n}$ (so this curve may contain complex points), which starts at $y$ and ends at a point $y^{\prime}$ in $D_{\mathbb{C}}^{n}$, and such that the moment map $\mathbf{F}$ is constant on this curve. This curve $\Gamma$ is not unique in general, but what's important is its existence. The (locally-defined) action variables $\left(H_{1}, \ldots, H_{n-1}\right)$ must be constant on $\Gamma$ too. Therefore the value of $\left(H_{1}, \ldots, H_{n-1}\right)$ at $y$, after the holonomy action of $\gamma$, becomes $\left(H_{1}\left(y^{\prime}\right), \ldots, H_{n-1}\left(y^{\prime}\right)\right)$. Since there are a finite number of points in $D_{\mathbb{C}}^{n}$ which have the same value of the moment map as $y$ (the number of points is the multiplicity of $\mathbf{F}$ at $x$ ), it follows easily that the holonomy is finite. We suspect that the holonomy is either trivial or $\mathbb{Z} / 2 \mathbb{Z}$ because the rank of $\mathbf{F}$ on $N$ is $(n-1)$, but we don't have yet a rigorous proof of this fact.

We have shown the existence of a $\mathbb{T}^{n-1}$-action for the different cases of Theorem 1.1. The fact that this action can be made free on Liouville tori follows from the following simple argument: if it is not free on a Liouville torus, then the corresponding isotropy group for this torus will also be the isotropy group for all nearby Liouville tori, and by analytic continuation this isotropy group will act trivially on a neighborhood of $N$. Therefore we can kill this isotropy group by taking the corresponding quotient group of $\mathbb{T}^{n-1}$. Theorem 1.1 is proved.

It would be nicer if we would not need a finite covering in the last case of Theorem 1.1. But the following example shows that sometimes it cannot be avoided.

Example. Consider the manifold $M^{4}=S^{1} \times D^{1} \times S^{1} \times D^{1}$ (where $S^{1}$ is a circle, $D^{1}=(-\epsilon,+\epsilon)$ is a real interval) with coordinates $(\alpha, H, \beta, Q)(\alpha$ and $\beta$ are cyclic coordinates and defined modulo 1 ), and with a standard symplectic form $\omega=d H \wedge d \alpha+d Q \wedge d \beta$. Let $G=G(\beta, Q)$ be an analytic function on $S^{1} \times D^{1}$ which satisfies the following conditions: 1$) G$ is invariant under the involution $(\beta, Q) \mapsto$ $(\beta+1 / 2, Q) ; 2) Q$ has exactly two critical points $(0,0)$ and $(1 / 2,0)$, and the value of $Q$ at these points is $0 ; 3)$ the singular level set $Q^{-1}(0)$ is homeomorphic but not diffeomorphic to a circle, and the nearby level sets are smooth circles. It is not difficult to construct such a function explicitly. Put $F_{1}=\cos (2 \pi \beta) H+G$ and $F_{2}=$ $H^{2}$. Then $\left(F_{1}, F_{2}\right)$ is the moment map of an integrable system. It is easy to see that the level set $N=\left\{F_{1}=F_{2}=0\right\}$ is a singular level set, which is a topological torus but not a smooth torus. $d F_{2}=0$ everywhere on $N$ but $d F_{1} \neq 0$ everywhere on $N$, so $N$ is a corank-one singularity. $F_{1}, F_{2}$ and the symplectic structure are invariant under the following free involution $\tau:(\alpha, H, \beta, Q) \mapsto(-\alpha,-H, \beta+1 / 2, Q)$. Taking quotient by this involution, we get an integrable system on $M^{4} / \tau$ with the moment map $\left(F_{1}, F_{2}\right)$. $H$, the would-be action variable corresponding to a Hamiltonian $\mathbb{S}^{1}$-action, will be double-valued there. 


\section{Generic degenerate singularities}

Let $\mathcal{O}(x)$ be a degenerate singular compact orbit of dimension $(n-1)$ for an integrable system with $n$ degrees of freedom, and let $D^{n+1}$ be a small ball transversal to $\mathcal{O}(x)$ at $x$. The germ of the integrable system at $\mathcal{O}(x)$ will be called a local corank-1 singularity of the integrable system. (The adjective "local" is used because in general $\mathcal{O}(x)$ is only a subset of a singular level set. When we consider singular level sets, as in [9] or Theorem 1.1, then we drop this adjective). Two local corank-1 singularities at $\mathcal{O}(x)$ and $\mathcal{O}^{\prime}\left(x^{\prime}\right)$ are called smoothly equivalent if there is a diffeomorphism from a neighborhood of $\mathcal{O}(x)$ to a neighborhood of $\mathcal{O}^{\prime}\left(x^{\prime}\right)$ which preserves the level sets of the moment map.

Let $D^{n+1}$ be a small ball transversal to $\mathcal{O}(x)$ at $x$, and let $\left(H_{1}, \ldots, H_{n-1}\right)$ be the action variables corresponding to the (essentially unique) Hamiltonian $\mathbb{T}^{n-1}$ action in a neighborhood of $\mathcal{O}(x)$. On $D^{n+1}$ we have a singular dimension-1 foliation by the level sets of the moment map. Since $H_{1}, \ldots, H_{n-1}$ are invariant on the level sets of the moment map, without the loss of generality we can assume that this singular foliation on $D^{n+1}$ is given by the level sets of $\left(H_{1}, \ldots, H_{n+1}, F_{n}\right)$ where $F_{n}$ is the last component of the moment map. In other words, without changing the topology of the integrable system (in a neighborhood of $\mathcal{O}(x)$ ), we can change the moment map to $\left(H_{1}, \ldots, H_{n-1}, F_{n}\right) . D^{n+1}$ can be considered as an $(n-1)$-dimensional family of the level sets of $\left(H_{1}, \ldots, H_{n-1}\right)$. These level sets are symplectic 2-dimensional disks, with the symplectic structure induced by the local Marsden-Weinstein reduction with respect to the action of $(n-1)$ commuting symplectic vector fields $X_{H_{1}}, \ldots, X_{H_{n-1}}$, and on them we have Hamiltonian systems with the Hamiltonian function $F_{n}$. Recall that the Hamiltonian $\mathbb{T}^{n-1}$-action is locally free, but it may be free or not. When this action is not free, it induces an action of a finite group (which is a quotient group of $\pi_{1}(\mathcal{O}(x))$ ) on $D^{n+1}$ which preserves $\left(H_{1}, \ldots, H_{n-1}, F_{n}\right)$ and the symplectic forms on the disks. It particular this finite group action preserves the orientation of the disks, and hence it is conjugate to the standard linear action of a cyclic group $\mathbb{Z} / m \mathbb{Z}$, and we have a surjective homomorphism from $\pi_{1}(\mathcal{O}(x))=\mathbb{Z}^{n-1}$ to $\mathbb{Z} / m \mathbb{Z}$.

The germ of the above $(n-1)$-dimensional family of $\mathbb{Z} / m \mathbb{Z}$-symmetric Hamiltonian systems with one degree of freedom will be called the reduced Hamiltonian system for the local singularity at $\mathcal{O}(x)$. It is essentially the Marsden-Weinstein reduction (the only difference is that, in case $m \neq 1$, the usual Marsden-Weinstein reduction will give orbifolds which are the quotient of our disks by the cyclic action). We have the following obvious

Proposition 5.1. The smooth equivalence class of a local degenerate corank-1 singularity is uniquely determined by the smooth equivalent class of its reduced system and a surjective morphism from $\mathbb{Z}^{n}$ to $\mathbb{Z} / m \mathbb{Z}$.

In other words, the topological study of local corank-1 singularities is the same 
as the study of families local 2-variables functions which are invariant under the standard cyclic action of $\mathbb{Z} / m \mathbb{Z}$ on the disk, or equivalently, the study of deformations of a $\mathbb{Z} / m \mathbb{Z}$-invariant singularity of a function of two variables. If the deformation is versal (see e.g. $[1,7]$ ), then we have a generic singularity. Thus, for a singularity of a 2-variable function to appear generically in a corank-1 singularity of an integrable system, the number of degrees of freedom $n$ must be greater than the dimension of the miniversal deformation of this singularity, and vice versa. For example, the singularity of type $A_{k}^{-}$(i.e. function $y^{2}-x^{k+1}$ with miniversal deformation $y^{2}-x^{k+1}+q_{1} x^{k-1}+q_{2} x^{k-2}+\ldots+q_{k}$, see e.g. [1]) will appear generically as a codimension 1 singularity whose torus $\mathbb{T}^{n-1}$ action is free for integrable systems with at least $k$ degrees of freedom. A (partial) classification of (isolated) singularities of real functions, due to Arnold et al., can be seen for example in [1]. Here we have simple singularities (A-D-E series), then unimodal, then bimodal singularities, etc.

The case with nontrivial cyclic action was also studied partially by various authors. For systems with 2 degrees of freedom, which amount to 1-parameter families of functions, generic corank-1 singularities have been studied by Golubitsky and Stewart [5], and also Kalashnikov [6]. The list of generic singularities in this case, taken from [5,6], is as follows (we will write them in the form of 1-parameter families of functions of two variables):

$m=2$ (i.e. $\mathbb{Z} / 2 \mathbb{Z}$ action): $F_{q}^{ \pm}(x, y)=x^{4} \pm y^{2}+q x^{2}$. (Notice that the case $x^{4}-y^{2}+q x^{2}$ was omitted in [5], but the sign here really matters, i.e. it will give different topological pictures).

$m=3: \Re z^{3}-q z \bar{z}$, where $z=x+i y$.

$m=4:(z \bar{z})^{2}+\gamma \Re z^{4}-q z \bar{z}$. Here $\gamma$ is a real modal parameter, $\gamma \neq 0, \pm 1$. Topologically there are 2 different cases, $0<|\gamma|<1$ and $1<|\gamma|<\infty$.

$m \geq 5:(z \bar{z})^{2}+\Re z^{m}-q z \bar{z}$.

For more-parameter families of $\mathbb{Z} / m \mathbb{Z}$-symmetric functions, we refer to a work of Wassermann [8].

\section{References}

[1] V. I. Arnold, V. A. Vasiliev, V. V. Goruynov and O. V. Lyashko, Singularities. Local and global theory. Dynamical Systems VI (V.I. Arnold ed.), Encyclopedia of Mathematical Sciences, Vol. 6, Springer-Verlag, (1993).

[2] M. Audin, The topology of torus actions on symplectic manifolds, Progress in mathematics, Vol. 93, Birkhäuser-Verlag, (1991).

[3] Tit Bau and Nguyen Tien Zung, Singularities of integrable and near integrable systems, Journal of Nonlinear Science 7 (1997), 1-7.

[4] A. T. Fomenko, Topological classification of all integrable Hamiltonian systems of general types with two degrees of freedom, MSRI Publications, 22 (1991), 131-340.

[5] M. Golubitsky and I. Stewart, Generic bifurcation of Hamiltonian systems with symmetry, Physica D 24 (1987), 391-405.

[6] V. Kalashnikov, A class of generic integrable Hamiltonian systems with two degrees of 
freedom, preprint 1996.

[7] V. Poènaru, Singularités $C^{\infty}$ en présence de symétrie, Lecture Notes in Mathematics, Vol. 510, 1976.

[8] G. Wassermann, Classification of singularities with compact Abelian symmetry, Banach Center Publications 20 (1988), 475-498.

[9] Nguyen Tien Zung, Symplectic topology of integrable Hamiltonian systems, I: ArnoldLiouville with singularities, Compositio Math. 101 (1996), 179-215; II: Characteristic classes and integrable surgery, preprint Max-Plank Insitut für Mathematik, 1996.

Nguyen Tien Zung

Dept. de Mathematiques

Université Montpellier II

Place Eugène Bataillon

F-34095 Montpellier Cedex 5

France

e-mail: tienzung@math.univ-montp2.fr

(Received: December 6, 1998) 